\title{
Amide-Silyl Ligand Exchanges and Equilibria among Group 4 Amide and Silyl Complexes
}

Hu Cai, ${ }^{\text {a } X i a n g h u a ~ Y u, ~}{ }^{a}$ Shujian Chen, ${ }^{\mathrm{a}}$ He Qiu, ${ }^{\mathrm{a}}$ Ilia A. Guzei, ${ }^{\mathrm{b}}$ Zi-Ling Xue ${ }^{\mathrm{a}}$

${ }^{a}$ Department of Chemistry, The University of Tennessee, Knoxville, Tennessee 37996-1600

${ }^{\mathrm{b}}$ Department of Chemistry, The University of Wisconsin, Madison, Wisconsin 53706-1396

Supporting Information 
$\mathrm{KNMe}_{2}$ is expected to be a product in the reaction in Eq. 7. It was prepared in order to obtain its NMR spectra. ${ }^{\mathrm{S} 1}$ In its preparation, toluene was first treated with a superbasic, 1:1 molar mixture of n-BuLi and $\mathrm{KOBu}^{\mathrm{t}}$ to yield $\mathrm{KCH}_{2} \mathrm{Ph}$ (Eq. S1). ${ }^{\mathrm{S} 2}$ This was followed by the addition of 1 equiv of $\mathrm{HNMe}_{2}$ to give $\mathrm{KNMe}_{2}$ (Eq. S2). The transmetallation in Eq. S1 is an exothermal reaction due to the formation of the strong $\mathrm{Li}-\mathrm{O}$ bond. $\mathrm{KNMe}_{2}$ was found to be pyrophoric and unstable in benzene- $d_{6}$ and THF- $d_{8}$, decomposing to unknown species.

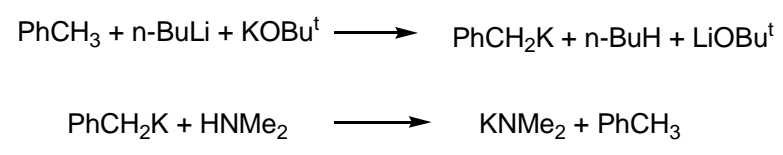

\section{Experimental}

Preparation of $\mathrm{KNMe}_{2}$. $\mathrm{n}$-BuLi (5.5 mL, 1.6 M in hexane; $8.8 \mathrm{mmol}$ ) was added dropwise to a suspension of $\mathrm{KOBu}^{\mathrm{t}}(1.0 \mathrm{~g}, 8.9 \mathrm{mmol})$ in toluene $(30 \mathrm{~mL})$ at $-30{ }^{\circ} \mathrm{C}$. A red solid was observed as a precipitate. After $2 \mathrm{~h}$, the precipitate was filtered. Then the red solid was washed with pentane $(20 \mathrm{~mL})$ twice. At $-30{ }^{\circ} \mathrm{C}, \mathrm{HNMe}_{2}(5.5 \mathrm{~mL}, 1.6 \mathrm{M}$ in THF, $8.8 \mathrm{mmol})$ was added to a suspension of the red solid in toluene $(30 \mathrm{~mL})$. The solution was stirred for another $2 \mathrm{~h}$. The red solid disappeared, and a white solid gradually formed during this time. The solid was washed with pentane to give a white solid of $\mathrm{KNMe}_{2}\left(655 \mathrm{mg}, 7.87 \mathrm{mmol}, 90 \%\right.$ yield). In ${ }^{1} \mathrm{H} \mathrm{NMR}$ in THF- $d_{8}$ at $23{ }^{\circ} \mathrm{C}$, a peak at $2.40 \mathrm{ppm}$ was observed. In ${ }^{13} \mathrm{C}\left\{{ }^{1} \mathrm{H}\right\}$ NMR at $23{ }^{\circ} \mathrm{C}$, a weak, broad peak at $41.0 \mathrm{ppm}$ was observed. During the ${ }^{13} \mathrm{C}$ data acquisition, $\mathrm{KNMe}_{2}$ in $\mathrm{THF}-\mathrm{d}_{8}$ decomposed to unknown species.

NMR assignments for $\mathrm{Zr}\left(\mathrm{NMe}_{2}\right)_{5}^{-}$(4a) in THF- $\boldsymbol{d}_{8}$. In a solution of crystals of 5 in THF- $d_{8}$ giving the equilibrium in Eq. 1, assignments of ${ }^{1} \mathrm{H}$ and ${ }^{13} \mathrm{C}$ NMR spectra of $\operatorname{Zr}\left(\mathrm{NMe}_{2}\right)_{5}{ }^{-}$(4a) in Eq. 1 were made, as discussed earlier. These assignments were supported by the following 
additional studies.

In the ${ }^{13} \mathrm{C}$ and ${ }^{1} \mathrm{H}$ spectra of a mixture $(\mathbf{A})$ of $\mathrm{Zr}\left(\mathrm{NMe}_{2}\right)_{4}(\mathbf{1 a}, 31 \mathrm{mg}, 0.12 \mathrm{mmol})$ and $\mathrm{LiNMe}_{2}$ (2.9 mg, $\left.0.057 \mathrm{mmol}\right)$ in THF- $d_{8}$, two peaks of ca. equal intensity $\left({ }^{1} \mathrm{H}: 2.89 \mathrm{ppm},{ }^{13} \mathrm{C}\right.$ : 44.74 ppm and ${ }^{1} \mathrm{H}$ : $2.81 \mathrm{ppm}, 43.11 \mathrm{ppm}$, respectively) were observed. The peaks at $2.89 \mathrm{ppm}$ in ${ }^{1} \mathrm{H}$ and $44.74 \mathrm{ppm}$ in ${ }^{13} \mathrm{C}$ NMR were assigned to those of 1a. When $3.2 \mathrm{mg}$ of $\mathrm{LiNMe}_{2}(0.063$ mmol; Total of the two portions: $0.120 \mathrm{mmol}$ ) was added to the mixture (B), only the peaks at $2.81 \mathrm{ppm}$ and $43.11 \mathrm{ppm}$ in ${ }^{1} \mathrm{H}$ and ${ }^{13} \mathrm{C}$ NMR, respectively, were observed. These were assigned to be those of $\mathrm{Zr}\left(\mathrm{NMe}_{2}\right)_{5}{ }^{-}$(4a).

When another portion of $\mathrm{LiNMe}_{2}(3.7 \mathrm{mg}, 0.073 \mathrm{mmol})$ was added to this mixture $(\mathbf{C})$, a new peak $\left({ }^{1} \mathrm{H}: 2.74 \mathrm{ppm},{ }^{13} \mathrm{C}\right.$ : $\left.46.39 \mathrm{ppm}\right)$ identified to be that of $\mathrm{Zr}\left(\mathrm{NMe}_{2}\right)_{6}{ }^{2-}$ (10) was observed along with that of $\mathrm{Zr}\left(\mathrm{NMe}_{2}\right)_{5}{ }^{-}$(4a) The results suggest that $\mathbf{A}$ is a mixture of $\mathbf{1 a}$ and $\mathrm{Zr}\left(\mathrm{NMe}_{2}\right)_{5}{ }^{-}$ (4a), and that $\mathbf{B}$ (molar ratio of $\mathbf{1 a} / \mathrm{LiNMe}_{2}=1$ ) is predominantly $\mathbf{4 a}$. With the addition of a total of 1.6 equiv of $\mathrm{LiNMe}_{2}$ to $\mathrm{Zr}\left(\mathrm{NMe}_{2}\right)_{4}$ (1a) in $\mathbf{C}$, it is a mixture of $\mathrm{Zr}\left(\mathrm{NMe}_{2}\right)_{5}{ }^{-}$(4a) and $\operatorname{Zr}\left(\mathrm{NMe}_{2}\right)_{6}{ }^{2-}(\mathbf{1 1})$

Formation of $\mathrm{Zr}\left(\mathrm{NMe}_{2}\right)_{\mathbf{4}}(\mathbf{T H F})_{2}$ in toluene- $\mathbf{d}_{\mathbf{8}}$. Bradley, Chisholm and co-workers have reported that, in benzene and toluene, $\mathrm{Zr}\left(\mathrm{NMe}_{2}\right)_{4}$ (1a) shows a degree of oligomerization. ${ }^{7,8 a, 21}$ For a mixture of 1a $(48.2 \mathrm{mg}, 0.180 \mathrm{mmol})$ and THF (8.9 mg, $0.12 \mathrm{mmol})$ in toluene- $d_{8}$ at $223 \mathrm{~K}$, the resonances of $\mathrm{Zr}\left(\mathrm{NMe}_{2}\right)_{4}(\mathrm{THF})_{2}$ was observed at 3.29 (THF), $2.98\left(\mathrm{NMe}_{2}\right)$ and 1.11 (THF) ppm in ${ }^{1} \mathrm{H}$ NMR spectrum, and 68.86 (THF), 43.36 (NMe $)$, and 25.64 (THF) ppm in ${ }^{13} \mathrm{C}$ NMR spectrum, respectively. In addition, the NMR resonances of the dimer of $\mathbf{1 a}^{8 \mathrm{a}}\left[{ }^{1} \mathrm{H}\right.$ NMR $\delta 2.88$ (terminal $-\mathrm{NM} e_{2}$ ), 2.34 (bridging $-\mathrm{NM} e_{2}$ ); ${ }^{13} \mathrm{C} \delta 43.76$ (terminal and bridging $-\mathrm{N} M e_{2}$ )] were observed as well. At $263 \mathrm{~K}$, the amide peaks in the ${ }^{1} \mathrm{H}$ NMR spectrum of the mixture of $\mathrm{Zr}\left(\mathrm{NMe}_{2}\right)_{4}(\mathrm{THF})_{2}$ and the dimer of 1a started to coalescence. At $296 \mathrm{~K}$, only one amide 
resonance was observed in the ${ }^{1} \mathrm{H}$ NMR spectrum.

\section{Additional References}

S1. $\quad \mathrm{KNMe}_{2}$ has been prepared from the reaction of $\mathrm{KNH}_{2}$ with $\mathrm{HNMe}_{2}$. Lambert, I.;

Ravoire, J. Ger. Offen. 1971, German Patent Application: DE 71-2117970 19710414. Chemical Abstracts No. 76:16262.

S2. Schlosser, M.; Hartmann, J. Angew. Chem. 1973, 85, 544. 


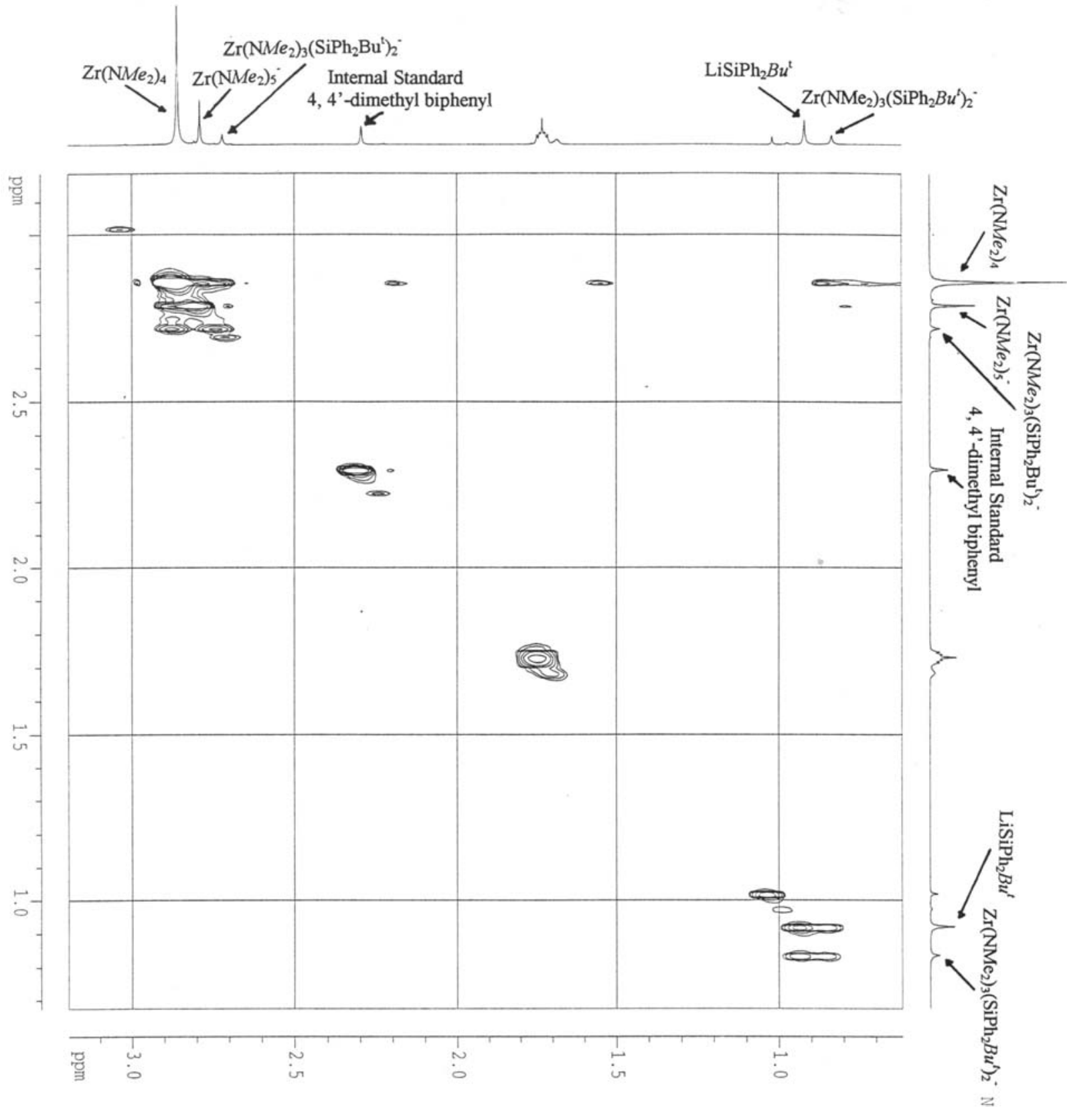

Figure S1. EXSY spectrum (THF- $d_{8}, 400.0 \mathrm{MHz}, 32{ }^{\circ} \mathrm{C}, t_{\text {mix }}=2 \mathrm{~s}$ ) of a mixture of $1 \mathbf{a}$ and 2. 


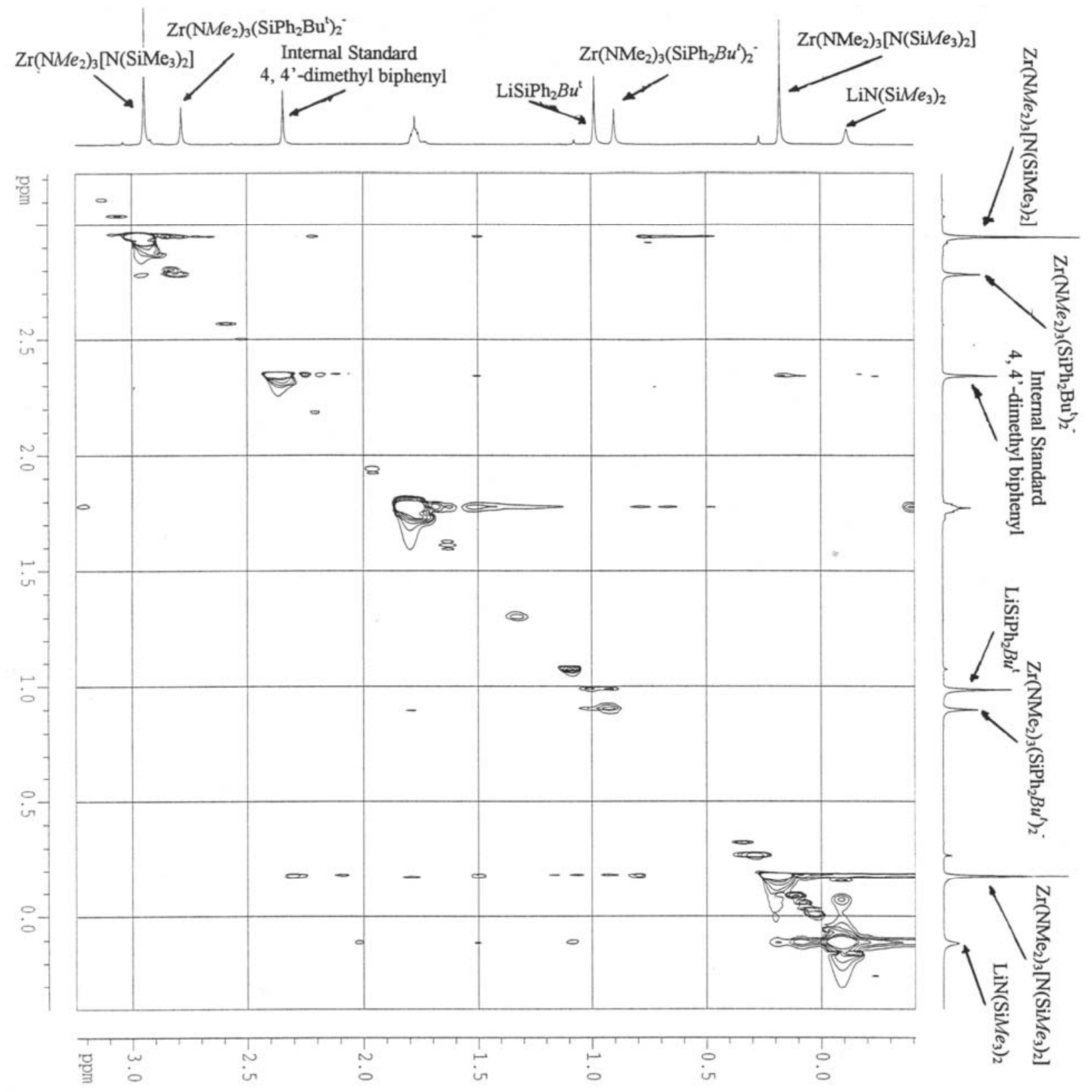

Figure S2. EXSY spectrum $\left(\mathrm{THF}-d_{8}, 400.0 \mathrm{MHz}, 32{ }^{\circ} \mathrm{C}, t_{\mathrm{mix}}=4 \mathrm{~s}\right)$ of a mixture of $\mathbf{6 a}$ and 2. EXSY spectrum (THF- $d_{8}, 32{ }^{\circ} \mathrm{C}$ ) with $t_{\text {mix }}=2 \mathrm{~s}$ did not show crosspeaks. Decomposition of the mixture was observed during the acquisition of the EXSY data at $32{ }^{\circ} \mathrm{C}$, making it difficult to conduct EXSY NMR studies at a higher temperature. 

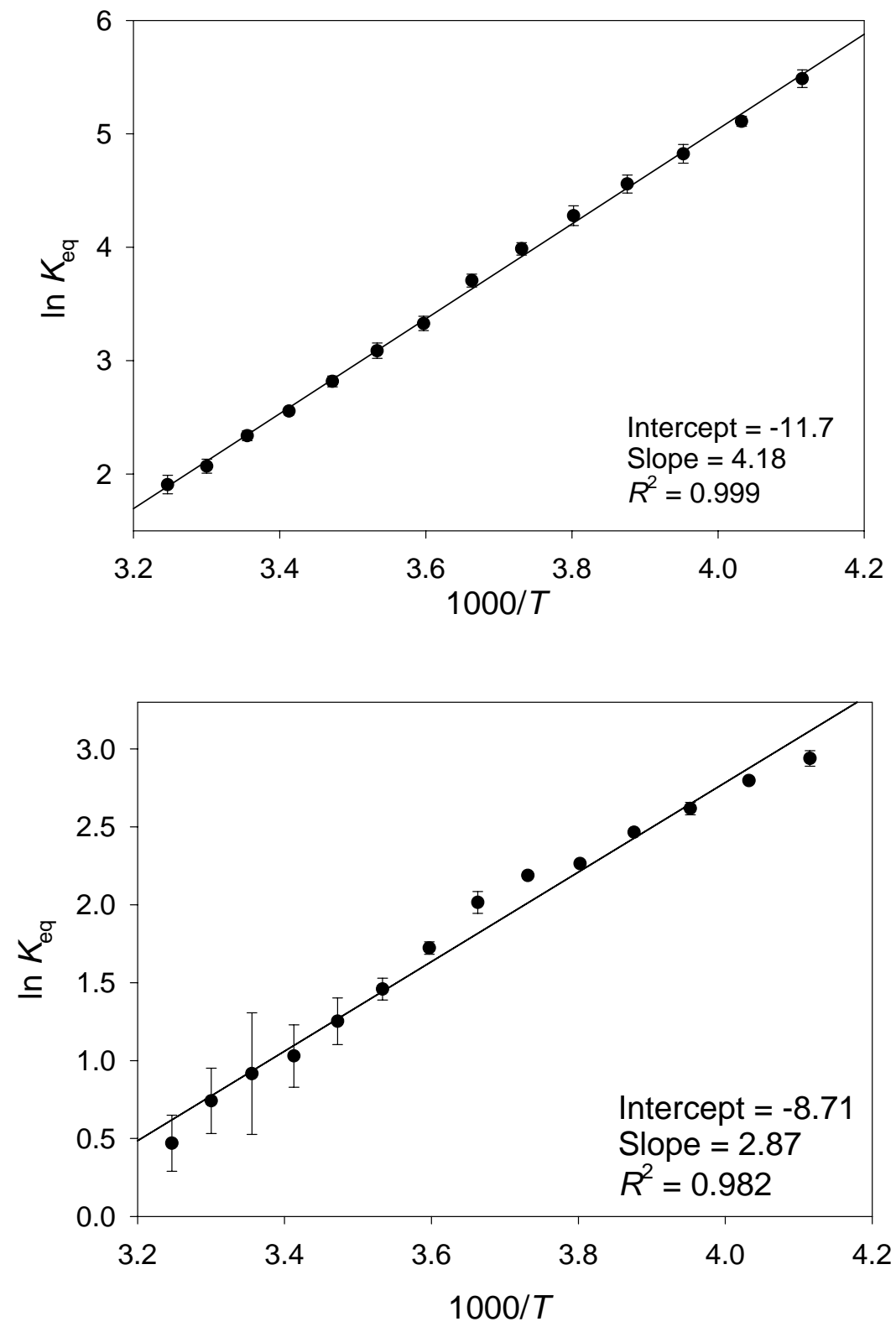

Figure S3. The $\ln K_{\text {eq }} v s$ 1000/T plots of the equilibria in Eq. 2: (top) Zr complexes; (bottom) Hf complexes. 


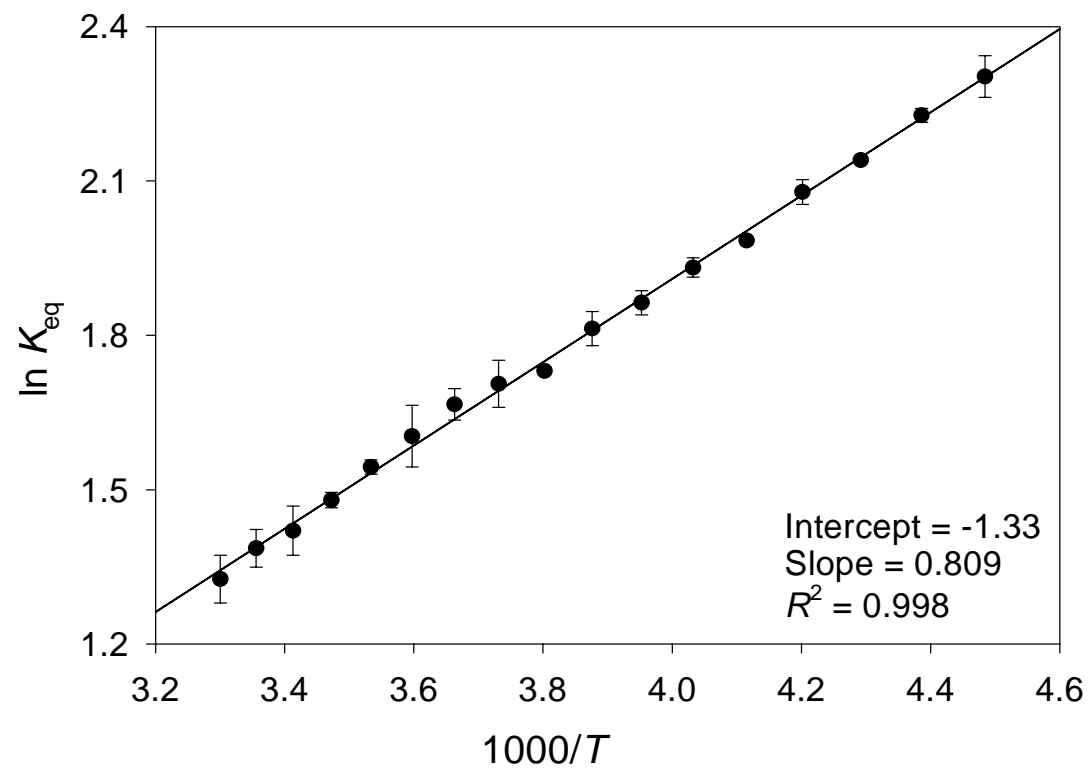

Figure S4. The $\ln K_{\text {eq }} v s$ 1000/T plot of the equilibrium in Eq. 4. 
Table S1. Crystal data and structure refinement for $\mathbf{5}$

\begin{tabular}{|c|c|c|}
\hline Empirical formula & \multicolumn{2}{|c|}{$\mathrm{C}_{64} \mathrm{H}_{118} \mathrm{Li}_{2} \mathrm{~N}_{8} \mathrm{O}_{2} \mathrm{Si}_{2} \mathrm{Zr}_{2}$} \\
\hline Formula weight & \multicolumn{2}{|c|}{1316.18} \\
\hline Temperature & \multicolumn{2}{|l|}{$173(2) \mathrm{K}$} \\
\hline Wavelength & \multicolumn{2}{|l|}{$0.71073 \AA$} \\
\hline Crystal system & \multicolumn{2}{|l|}{ Monoclinic } \\
\hline Space group & \multicolumn{2}{|l|}{$\mathrm{C} 2 / \mathrm{c}$} \\
\hline \multirow[t]{3}{*}{ Unit cell dimensions } & $a=25.145(3) \AA$ & $\alpha=90^{\circ}$ \\
\hline & $b=17.770(3) \AA$ & $\beta=97.789(3)^{\circ}$ \\
\hline & $c=16.346(3) \AA$ & $\gamma=90^{\circ}$ \\
\hline Volume & \multicolumn{2}{|l|}{$7236(2) \AA^{3}$} \\
\hline Z & \multicolumn{2}{|l|}{4} \\
\hline Density (calculated) & \multicolumn{2}{|l|}{$1.208 \mathrm{~g} / \mathrm{cm}^{3}$} \\
\hline Absorption coefficient & \multicolumn{2}{|l|}{$0.368 \mathrm{~mm}^{-1}$} \\
\hline$F(000)$ & \multicolumn{2}{|l|}{2816} \\
\hline Crystal size & \multicolumn{2}{|c|}{$0.40 \times 0.25 \times 0.25 \mathrm{~mm}^{3}$} \\
\hline Theta range for data collection & \multicolumn{2}{|c|}{1.41 to $28.28^{\circ}$} \\
\hline Index ranges & \multicolumn{2}{|c|}{$-32 \leq h \leq 33,-23 \leq k \leq 23,-21 \leq l \leq 21$} \\
\hline Reflections collected & \multicolumn{2}{|c|}{36482} \\
\hline Independent reflections & \multicolumn{2}{|c|}{$8684[R($ int $)=0.0543]$} \\
\hline Completeness to theta $=28.28^{\circ}$ & \multicolumn{2}{|c|}{$96.5 \%$} \\
\hline Absorption correction & \multicolumn{2}{|c|}{ Multi-scan with SADABS } \\
\hline Max. and min. transmission & \multicolumn{2}{|c|}{0.9136 and 0.8667} \\
\hline Refinement method & \multicolumn{2}{|c|}{ Full-matrix least-squares on $F^{2}$} \\
\hline Data / restraints / parameters & \multicolumn{2}{|c|}{8684 / 0 / 383} \\
\hline Goodness-of-fit on $F^{2}$ & \multicolumn{2}{|c|}{1.041} \\
\hline Final $R$ indices $[I>2 \operatorname{sigma}(I)]$ & \multicolumn{2}{|c|}{$R 1=0.0396, \mathrm{w} R 2=0.1030$} \\
\hline$R$ indices (all data) & \multicolumn{2}{|c|}{$R 1=0.0567, \mathrm{w} R 2=0.1169$} \\
\hline Largest diff. peak and hole & \multicolumn{2}{|c|}{0.875 and -0.464 e..$\AA^{-3}$} \\
\hline
\end{tabular}


Table S2. Atomic coordinates $\left(\times 10^{4}\right)$ and equivalent isotropic displacement parameters $\left(\AA^{2} \times\right.$ $10^{3}$ ) for 5 . $\mathrm{U}(\mathrm{eq})$ is defined as one third of the trace of the orthogonalized $\mathrm{U}^{\mathrm{ij}}$ tensor.

\begin{tabular}{|c|c|c|c|c|}
\hline & $\mathrm{x}$ & $\mathrm{y}$ & $\mathrm{z}$ & $\mathrm{U}(\mathrm{eq})$ \\
\hline $\operatorname{Zr}(1)$ & 0 & $1532(1)$ & 2500 & $21(1)$ \\
\hline $\operatorname{Zr}(2)$ & 5000 & $1512(1)$ & 2500 & $24(1)$ \\
\hline $\operatorname{Si}(1)$ & 1171(1) & $1670(1)$ & 2965(1) & $26(1)$ \\
\hline $\mathrm{O}(1)$ & $3564(1)$ & 2192(1) & $1107(1)$ & $49(1)$ \\
\hline $\mathrm{O}(2)$ & $3540(1)$ & $516(1)$ & $663(1)$ & $49(1)$ \\
\hline $\mathrm{N}(1)$ & 0 & $2702(1)$ & 2500 & $29(1)$ \\
\hline $\mathrm{N}(2)$ & $50(1)$ & $945(1)$ & $1434(1)$ & $32(1)$ \\
\hline $\mathrm{N}(3)$ & 5000 & 2658(2) & 2500 & $34(1)$ \\
\hline $\mathrm{N}(4)$ & $4821(1)$ & $1430(1)$ & $1121(1)$ & $35(1)$ \\
\hline $\mathrm{N}(5)$ & $4251(1)$ & 951(1) & 2513(1) & $34(1)$ \\
\hline $\operatorname{Li}(1)$ & $4012(2)$ & $1276(2)$ & 1271(3) & $37(1)$ \\
\hline $\mathrm{C}(1)$ & 232(1) & $3162(1)$ & $3187(2)$ & $38(1)$ \\
\hline$C(2)$ & $98(1)$ & $1342(2)$ & $678(2)$ & $42(1)$ \\
\hline C(3) & $5(1)$ & $139(2)$ & 1298(2) & $51(1)$ \\
\hline$C(4)$ & 1353(1) & $1680(1)$ & $4145(1)$ & $32(1)$ \\
\hline$C(5)$ & $1322(1)$ & $1013(2)$ & $4602(2)$ & $43(1)$ \\
\hline$C(6)$ & $1402(1)$ & $1004(2)$ & $5461(2)$ & $52(1)$ \\
\hline$C(7)$ & $1517(1)$ & $1655(2)$ & $5901(2)$ & $56(1)$ \\
\hline C(8) & $1545(1)$ & $2321(2)$ & $5473(2)$ & $59(1)$ \\
\hline $\mathrm{C}(9)$ & $1458(1)$ & 2332(2) & $4616(2)$ & $46(1)$ \\
\hline$C(10)$ & $1479(1)$ & 2582(1) & 2599(1) & $29(1)$ \\
\hline $\mathrm{C}(11)$ & $1947(1)$ & 2938(2) & $2975(2)$ & $38(1)$ \\
\hline $\mathrm{C}(12)$ & $2135(1)$ & $3607(2)$ & $2666(2)$ & $48(1)$ \\
\hline$C(13)$ & $1867(1)$ & $3930(2)$ & 1961(2) & $47(1)$ \\
\hline$C(14)$ & $1413(1)$ & $3587(1)$ & $1564(2)$ & $40(1)$ \\
\hline$C(15)$ & $1223(1)$ & 2931(1) & 1883(1) & $32(1)$ \\
\hline$C(16)$ & $1640(1)$ & $910(1)$ & $2561(2)$ & $35(1)$ \\
\hline $\mathrm{C}(17)$ & 2223(1) & $967(2)$ & $2975(2)$ & $55(1)$ \\
\hline $\mathrm{C}(18)$ & $1629(1)$ & 1039(2) & $1634(2)$ & $43(1)$ \\
\hline C(19) & $1429(1)$ & 113(2) & 2672(2) & $58(1)$ \\
\hline$C(20)$ & $4554(1)$ & $3120(2)$ & 2656(3) & $68(1)$ \\
\hline
\end{tabular}




\begin{tabular}{lrrrr}
$\mathrm{C}(21)$ & $4906(1)$ & $743(2)$ & $672(2)$ & $54(1)$ \\
$\mathrm{C}(22)$ & $4924(1)$ & $2059(2)$ & $591(2)$ & $53(1)$ \\
$\mathrm{C}(23)$ & $4248(1)$ & $127(2)$ & $2474(2)$ & $57(1)$ \\
$\mathrm{C}(24)$ & $3880(1)$ & $1204(2)$ & $3069(2)$ & $50(1)$ \\
$\mathrm{C}(25)$ & $2982(1)$ & $593(2)$ & $677(3)$ & $86(1)$ \\
$\mathrm{C}(26)$ & $2700(1)$ & $-5(2)$ & $166(2)$ & $63(1)$ \\
$\mathrm{C}(27)$ & $3124(1)$ & $-569(2)$ & $77(3)$ & $70(1)$ \\
$\mathrm{C}(28)$ & $3637(1)$ & $-204(2)$ & $312(3)$ & $83(1)$ \\
$\mathrm{C}(29)$ & $3568(2)$ & $2640(2)$ & $371(3)$ & $82(1)$ \\
$\mathrm{C}(30)$ & $3368(3)$ & $3369(3)$ & $514(3)$ & $113(2)$ \\
$\mathrm{C}(31)$ & $3063(2)$ & $3290(3)$ & $1206(3)$ & $108(2)$ \\
$\mathrm{C}(32)$ & $3160(1)$ & $2513(2)$ & $1548(2)$ & $56(1)$ \\
\hline
\end{tabular}


Table S3. Bond lengths $(\AA)$ and angles $\left({ }^{\circ}\right)$ for 5

\begin{tabular}{|c|c|c|c|}
\hline $\operatorname{Zr}(1)-N(2)$ & 2.0488(19) & $N(4)-C(21)$ & $1.454(3)$ \\
\hline $\mathrm{Zr}(1)-\mathrm{N}(2) \# 1$ & $2.0488(19)$ & $\mathrm{N}(4)-\mathrm{C}(22)$ & $1.459(3)$ \\
\hline $\mathrm{Zr}(1)-\mathrm{N}(1)$ & $2.080(2)$ & N(4)-Li(1) & $2.099(4)$ \\
\hline $\operatorname{Zr}(1)-\operatorname{Si}(1) \# 1$ & $2.9507(7)$ & $N(5)-C(24)$ & $1.459(3)$ \\
\hline $\operatorname{Zr}(1)-\operatorname{Si}(1)$ & $2.9507(7)$ & $\mathrm{N}(5)-\mathrm{C}(23)$ & $1.465(3)$ \\
\hline $\mathrm{Zr}(2)-\mathrm{N}(3)$ & $2.036(3)$ & $\mathrm{N}(5)-\operatorname{Li}(1)$ & $2.117(5)$ \\
\hline $\operatorname{Zr}(2)-N(5)$ & 2.1337(19) & $\mathrm{C}(4)-\mathrm{C}(9)$ & $1.397(4)$ \\
\hline Zr(2)-N(5)\#2 & 2.1337(19) & $C(4)-C(5)$ & $1.408(3)$ \\
\hline $\operatorname{Zr}(2)-\mathrm{N}(4)$ & $2.243(2)$ & $\mathrm{C}(5)-\mathrm{C}(6)$ & $1.392(4)$ \\
\hline Zr(2)-N(4)\#2 & $2.243(2)$ & $\mathrm{C}(6)-\mathrm{C}(7)$ & $1.373(5)$ \\
\hline $\operatorname{Zr}(2)-\operatorname{Li}(1) \# 2$ & $3.005(4)$ & $C(7)-C(8)$ & $1.380(5)$ \\
\hline $\operatorname{Zr}(2)-\operatorname{Li}(1)$ & $3.005(4)$ & $\mathrm{C}(8)-\mathrm{C}(9)$ & $1.389(4)$ \\
\hline Si(1)-C(4) & $1.920(2)$ & $\mathrm{C}(10)-\mathrm{C}(15)$ & $1.400(3)$ \\
\hline $\operatorname{Si}(1)-C(10)$ & $1.926(2)$ & $C(10)-C(11)$ & $1.403(3)$ \\
\hline $\mathrm{Si}(1)-\mathrm{C}(16)$ & $1.965(2)$ & $\mathrm{C}(11)-\mathrm{C}(12)$ & $1.399(4)$ \\
\hline $\mathrm{O}(1)-\mathrm{C}(32)$ & $1.440(3)$ & $C(12)-C(13)$ & $1.379(4)$ \\
\hline $\mathrm{O}(1)-\mathrm{C}(29)$ & $1.442(4)$ & $\mathrm{C}(13)-\mathrm{C}(14)$ & $1.375(4)$ \\
\hline $\mathrm{O}(1)-\mathrm{Li}(1)$ & $1.979(5)$ & $C(14)-C(15)$ & $1.388(3)$ \\
\hline $\mathrm{O}(2)-\mathrm{C}(25)$ & $1.413(3)$ & $C(16)-C(18)$ & $1.529(4)$ \\
\hline $\mathrm{O}(2)-\mathrm{C}(28)$ & $1.437(3)$ & $\mathrm{C}(16)-\mathrm{C}(17)$ & $1.531(4)$ \\
\hline $\mathrm{O}(2)-\mathrm{Li}(1)$ & $1.975(5)$ & $\mathrm{C}(16)-\mathrm{C}(19)$ & $1.531(4)$ \\
\hline $\mathrm{N}(1)-\mathrm{C}(1) \# 1$ & $1.447(3)$ & $C(25)-C(26)$ & $1.471(4)$ \\
\hline $\mathrm{N}(1)-\mathrm{C}(1)$ & $1.447(3)$ & $\mathrm{C}(26)-\mathrm{C}(27)$ & $1.485(5)$ \\
\hline $\mathrm{N}(2)-\mathrm{C}(2)$ & $1.442(3)$ & $C(27)-C(28)$ & $1.450(5)$ \\
\hline$N(2)-C(3)$ & $1.452(3)$ & $C(29)-C(30)$ & $1.420(5)$ \\
\hline N(3)-C(20)\#2 & $1.440(3)$ & $\mathrm{C}(30)-\mathrm{C}(31)$ & $1.456(6)$ \\
\hline $\mathrm{N}(3)-\mathrm{C}(20)$ & $1.440(3)$ & $C(31)-C(32)$ & $1.497(5)$ \\
\hline$N(2)-\operatorname{Zr}(1)-N(2) \# 1$ & $118.86(11)$ & $N(2)-\operatorname{Zr}(1)-\operatorname{Si}(1)$ & $95.01(6)$ \\
\hline N(2)-Zr(1)-N(1) & $120.57(6)$ & $\mathrm{N}(2) \# 1-\operatorname{Zr}(1)-\operatorname{Si}(1)$ & 89.83(6) \\
\hline $\mathrm{N}(2) \# 1-\operatorname{Zr}(1)-\mathrm{N}(1)$ & $120.57(6)$ & $\mathrm{N}(1)-\operatorname{Zr}(1)-\mathrm{Si}(1)$ & $85.235(13)$ \\
\hline $\mathrm{N}(2)-\operatorname{Zr}(1)-\operatorname{Si}(1) \# 1$ & 89.83(6) & $\operatorname{Si}(1) \# 1-\operatorname{Zr}(1)-\operatorname{Si}(1)$ & $170.47(3)$ \\
\hline $\mathrm{N}(2) \# 1-\operatorname{Zr}(1)-\mathrm{Si}(1) \# 1$ & 95.01(6) & $\mathrm{N}(3)-\operatorname{Zr}(2)-\mathrm{N}(5)$ & $117.85(5)$ \\
\hline N(1)-Zr(1)-Si(1)\#1 & $85.235(13)$ & N(3)-Zr(2)-N(5)\#2 & $117.85(5)$ \\
\hline
\end{tabular}




\begin{tabular}{|c|c|c|c|}
\hline N(5)-Zr(2)-N(5)\#2 & 124.30(11) & $\mathrm{C}(2)-\mathrm{N}(2)-\mathrm{Zr}(1)$ & $120.17(16)$ \\
\hline N(3)-Zr(2)-N(4) & 93.73(5) & $\mathrm{C}(3)-\mathrm{N}(2)-\mathrm{Zr}(1)$ & $128.32(17)$ \\
\hline N(5)-Zr(2)-N(4) & $85.54(8)$ & C(20)\#2-N(3)-C(20) & 110.4(3) \\
\hline N(5)\#2-Zr(2)-N(4) & $90.97(8)$ & $\mathrm{C}(20) \# 2-\mathrm{N}(3)-\mathrm{Zr}(2)$ & $124.79(16)$ \\
\hline $\mathrm{N}(3)-\operatorname{Zr}(2)-\mathrm{N}(4) \# 2$ & 93.73(5) & $\mathrm{C}(20)-\mathrm{N}(3)-\mathrm{Zr}(2)$ & $124.79(16)$ \\
\hline $\mathrm{N}(5)-\operatorname{Zr}(2)-\mathrm{N}(4) \# 2$ & $90.97(8)$ & $\mathrm{C}(21)-\mathrm{N}(4)-\mathrm{C}(22)$ & $107.0(2)$ \\
\hline $\mathrm{N}(5) \# 2-\mathrm{Zr}(2)-\mathrm{N}(4) \# 2$ & $85.54(8)$ & $\mathrm{C}(21)-\mathrm{N}(4)-\mathrm{Li}(1)$ & $99.3(2)$ \\
\hline N(4)-Zr(2)-N(4)\#2 & $172.54(11)$ & $\mathrm{C}(22)-\mathrm{N}(4)-\mathrm{Li}(1)$ & $115.0(2)$ \\
\hline N(3)-Zr(2)-Li(1)\#2 & 98.03(8) & $C(21)-N(4)-\operatorname{Zr}(2)$ & $122.56(18)$ \\
\hline N(5)-Zr(2)-Li(1)\#2 & $125.38(10)$ & $\mathrm{C}(22)-\mathrm{N}(4)-\mathrm{Zr}(2)$ & $121.29(17)$ \\
\hline N(5)\#2-Zr(2)-Li(1)\#2 & $44.80(10)$ & $\mathrm{Li}(1)-\mathrm{N}(4)-\operatorname{Zr}(2)$ & 87.51(14) \\
\hline N(4)-Zr(2)-Li(1)\#2 & $134.27(10)$ & $\mathrm{C}(24)-\mathrm{N}(5)-\mathrm{C}(23)$ & $109.6(2)$ \\
\hline $\mathrm{N}(4) \# 2-\operatorname{Zr}(2)-\operatorname{Li}(1) \# 2$ & 44.26(9) & C(24)-N(5)-Li(1) & $113.0(2)$ \\
\hline N(3)-Zr(2)-Li(1) & $98.03(8)$ & C(23)-N(5)-Li(1) & $103.4(2)$ \\
\hline$N(5)-\operatorname{Zr}(2)-\operatorname{Li}(1)$ & $44.80(10)$ & $\mathrm{C}(24)-\mathrm{N}(5)-\mathrm{Zr}(2)$ & $120.11(18)$ \\
\hline N(5)\#2-Zr(2)-Li(1) & $125.38(10)$ & $\mathrm{C}(23)-\mathrm{N}(5)-\mathrm{Zr}(2)$ & $117.76(17)$ \\
\hline N(4)-Zr(2)-Li(1) & $44.26(9)$ & Li(1)-N(5)-Zr(2) & 89.96(13) \\
\hline N(4)\#2-Zr(2)-Li(1) & $134.27(10)$ & $\mathrm{O}(2)-\mathrm{Li}(1)-\mathrm{O}(1)$ & $101.6(2)$ \\
\hline Li(1)\#2-Zr(2)-Li(1) & 163.93(16) & $\mathrm{O}(2)-\mathrm{Li}(1)-\mathrm{N}(4)$ & 123.8(2) \\
\hline C(4)-Si(1)-C(10) & $104.70(10)$ & $\mathrm{O}(1)-\mathrm{Li}(1)-\mathrm{N}(4)$ & $114.8(2)$ \\
\hline C(4)-Si(1)-C(16) & 105.79(10) & $\mathrm{O}(2)-\mathrm{Li}(1)-\mathrm{N}(5)$ & $111.7(2)$ \\
\hline $\mathrm{C}(10)-\mathrm{Si}(1)-\mathrm{C}(16)$ & $100.95(10)$ & $\mathrm{O}(1)-\mathrm{Li}(1)-\mathrm{N}(5)$ & $115.9(2)$ \\
\hline C(4)-Si(1)-Zr(1) & $110.65(7)$ & N(4)-Li(1)-N(5) & 89.68(18) \\
\hline $\mathrm{C}(10)-\operatorname{Si}(1)-\operatorname{Zr}(1)$ & 114.71(7) & $\mathrm{O}(2)-\operatorname{Li}(1)-\operatorname{Zr}(2)$ & 144.9(2) \\
\hline C(16)-Si(1)-Zr(1) & 118.66(8) & $\mathrm{O}(1)-\operatorname{Li}(1)-\operatorname{Zr}(2)$ & $112.35(18)$ \\
\hline $\mathrm{C}(32)-\mathrm{O}(1)-\mathrm{C}(29)$ & $106.5(2)$ & N(4)-Li(1)-Zr(2) & $48.23(10)$ \\
\hline C(32)-O(1)-Li(1) & 133.2(2) & N(5)-Li(1)-Zr(2) & $45.24(10)$ \\
\hline C(29)-O(1)-Li(1) & 119.9(2) & C(9)-C(4)-C(5) & 115.1(2) \\
\hline $\mathrm{C}(25)-\mathrm{O}(2)-\mathrm{C}(28)$ & $108.4(2)$ & $\mathrm{C}(9)-\mathrm{C}(4)-\mathrm{Si}(1)$ & $124.25(19)$ \\
\hline C(25)-O(2)-Li(1) & $117.2(2)$ & $C(5)-C(4)-S i(1)$ & $120.18(19)$ \\
\hline C(28)-O(2)-Li(1) & $133.3(2)$ & $C(6)-C(5)-C(4)$ & $122.4(3)$ \\
\hline C(1)\#1-N(1)-C(1) & $111.2(3)$ & $C(7)-C(6)-C(5)$ & 120.6(3) \\
\hline $\mathrm{C}(1) \# 1-\mathrm{N}(1)-\mathrm{Zr}(1)$ & $124.40(13)$ & $C(6)-C(7)-C(8)$ & 118.6(3) \\
\hline C(1)-N(1)-Zr(1) & $124.40(13)$ & $\mathrm{C}(7)-\mathrm{C}(8)-\mathrm{C}(9)$ & $120.8(3)$ \\
\hline $\mathrm{C}(2)-\mathrm{N}(2)-\mathrm{C}(3)$ & $111.4(2)$ & $\mathrm{C}(8)-\mathrm{C}(9)-\mathrm{C}(4)$ & $122.5(3)$ \\
\hline
\end{tabular}




$\begin{array}{llll}\mathrm{C}(15)-\mathrm{C}(10)-\mathrm{C}(11) & 115.4(2) & \mathrm{C}(18)-\mathrm{C}(16)-\mathrm{Si}(1) & 107.36(16) \\ \mathrm{C}(15)-\mathrm{C}(10)-\mathrm{Si}(1) & 118.34(17) & \mathrm{C}(17)-\mathrm{C}(16)-\mathrm{Si}(1) & 112.81(18) \\ \mathrm{C}(11)-\mathrm{C}(10)-\mathrm{Si}(1) & 126.25(18) & \mathrm{C}(19)-\mathrm{C}(16)-\mathrm{Si}(1) & 111.21(18) \\ \mathrm{C}(12)-\mathrm{C}(11)-\mathrm{C}(10) & 122.0(3) & \mathrm{O}(2)-\mathrm{C}(25)-\mathrm{C}(26) & 108.9(3) \\ \mathrm{C}(13)-\mathrm{C}(12)-\mathrm{C}(11) & 120.2(3) & \mathrm{C}(25)-\mathrm{C}(26)-\mathrm{C}(27) & 104.2(3) \\ \mathrm{C}(14)-\mathrm{C}(13)-\mathrm{C}(12) & 119.4(2) & \mathrm{C}(28)-\mathrm{C}(27)-\mathrm{C}(26) & 107.3(3) \\ \mathrm{C}(13)-\mathrm{C}(14)-\mathrm{C}(15) & 120.1(3) & \mathrm{O}(2)-\mathrm{C}(28)-\mathrm{C}(27) & 108.0(3) \\ \mathrm{C}(14)-\mathrm{C}(15)-\mathrm{C}(10) & 122.9(2) & \mathrm{C}(30)-\mathrm{C}(29)-\mathrm{O}(1) & 108.9(3) \\ \mathrm{C}(18)-\mathrm{C}(16)-\mathrm{C}(17) & 108.4(2) & \mathrm{C}(29)-\mathrm{C}(30)-\mathrm{C}(31) & 105.9(4) \\ \mathrm{C}(18)-\mathrm{C}(16)-\mathrm{C}(19) & 107.2(2) & \mathrm{C}(30)-\mathrm{C}(31)-\mathrm{C}(32) & 107.7(3) \\ \mathrm{C}(17)-\mathrm{C}(16)-\mathrm{C}(19) & 109.6(2) & \mathrm{O}(1)-\mathrm{C}(32)-\mathrm{C}(31) & 105.5(3)\end{array}$

Symmetry transformations used to generate equivalent atoms:

$\# 1$-x,y,-z+1/2 \#2 -x+1,y,-z+1/2 
Table S4. Anisotropic displacement parameters $\left(\AA^{2} \times 10^{3}\right)$ for 5. The anisotropic displacement factor exponent takes the form: $-2 \pi^{2}\left[h^{2} a^{* 2} U^{11}+\ldots+2 h k a * b * U^{12}\right]$

\begin{tabular}{|c|c|c|c|c|c|c|}
\hline & $\mathrm{U}^{11}$ & $\mathrm{U}^{22}$ & $\mathrm{U}^{33}$ & $\mathrm{U}^{23}$ & $\mathrm{U}^{13}$ & $\mathrm{U}^{12}$ \\
\hline $\operatorname{Zr}(1)$ & $26(1)$ & $17(1)$ & $21(1)$ & 0 & $5(1)$ & 0 \\
\hline $\operatorname{Zr}(2)$ & $27(1)$ & $19(1)$ & $27(1)$ & 0 & $8(1)$ & 0 \\
\hline $\operatorname{Si}(1)$ & $26(1)$ & $26(1)$ & $25(1)$ & $1(1)$ & $4(1)$ & $3(1)$ \\
\hline $\mathrm{O}(1)$ & $56(1)$ & $40(1)$ & $55(1)$ & $-2(1)$ & $16(1)$ & $15(1)$ \\
\hline $\mathrm{O}(2)$ & $35(1)$ & $47(1)$ & $64(1)$ & $-23(1)$ & $1(1)$ & $1(1)$ \\
\hline $\mathrm{N}(1)$ & $32(1)$ & $17(1)$ & $39(2)$ & 0 & $6(1)$ & 0 \\
\hline $\mathrm{N}(2)$ & $37(1)$ & $32(1)$ & $28(1)$ & $-6(1)$ & $5(1)$ & $2(1)$ \\
\hline $\mathrm{N}(3)$ & $41(2)$ & $22(1)$ & $41(2)$ & 0 & $8(1)$ & 0 \\
\hline $\mathrm{N}(4)$ & $36(1)$ & $40(1)$ & $30(1)$ & $-2(1)$ & $10(1)$ & $1(1)$ \\
\hline $\mathrm{N}(5)$ & $34(1)$ & $31(1)$ & $39(1)$ & $0(1)$ & $11(1)$ & $-6(1)$ \\
\hline $\operatorname{Li}(1)$ & $32(2)$ & $34(2)$ & $44(2)$ & $-6(2)$ & $6(2)$ & $0(2)$ \\
\hline $\mathrm{C}(1)$ & $38(1)$ & $29(1)$ & $47(2)$ & $-7(1)$ & $8(1)$ & $0(1)$ \\
\hline $\mathrm{C}(2)$ & $45(2)$ & $48(2)$ & $34(1)$ & $0(1)$ & $8(1)$ & $-5(1)$ \\
\hline $\mathrm{C}(3)$ & $72(2)$ & $32(1)$ & $50(2)$ & $-8(1)$ & $12(1)$ & $3(1)$ \\
\hline$C(4)$ & $27(1)$ & $40(1)$ & $27(1)$ & $2(1)$ & $4(1)$ & $6(1)$ \\
\hline$C(5)$ & $50(2)$ & $46(2)$ & $34(1)$ & $6(1)$ & $6(1)$ & $7(1)$ \\
\hline$C(6)$ & $56(2)$ & $67(2)$ & $35(2)$ & $18(1)$ & $11(1)$ & $22(1)$ \\
\hline$C(7)$ & $51(2)$ & $92(2)$ & $26(1)$ & $1(2)$ & $6(1)$ & $23(2)$ \\
\hline $\mathrm{C}(8)$ & $72(2)$ & $70(2)$ & $36(2)$ & $-15(1)$ & $11(1)$ & $-1(2)$ \\
\hline $\mathrm{C}(9)$ & $59(2)$ & $47(2)$ & $34(1)$ & $-4(1)$ & $14(1)$ & $1(1)$ \\
\hline$C(10)$ & $28(1)$ & $31(1)$ & $28(1)$ & $-3(1)$ & $9(1)$ & $2(1)$ \\
\hline $\mathrm{C}(11)$ & $30(1)$ & $45(1)$ & $37(1)$ & $-3(1)$ & $5(1)$ & $-2(1)$ \\
\hline $\mathrm{C}(12)$ & $38(1)$ & $50(2)$ & $57(2)$ & $-11(1)$ & $14(1)$ & $-16(1)$ \\
\hline $\mathrm{C}(13)$ & $51(2)$ & $34(1)$ & $59(2)$ & $2(1)$ & $23(1)$ & $-6(1)$ \\
\hline$C(14)$ & $44(1)$ & $36(1)$ & $42(1)$ & $8(1)$ & $15(1)$ & $5(1)$ \\
\hline$C(15)$ & $32(1)$ & $33(1)$ & $32(1)$ & $2(1)$ & $6(1)$ & $0(1)$ \\
\hline$C(16)$ & $36(1)$ & $35(1)$ & $34(1)$ & $-1(1)$ & $5(1)$ & $10(1)$ \\
\hline$C(17)$ & $39(2)$ & $73(2)$ & $52(2)$ & $-5(2)$ & $0(1)$ & $27(1)$ \\
\hline$C(18)$ & $49(2)$ & $45(2)$ & $35(1)$ & $-6(1)$ & $10(1)$ & $12(1)$ \\
\hline \multirow[t]{2}{*}{ C(19) } & $82(2)$ & $33(1)$ & $64(2)$ & $2(1)$ & $28(2)$ & $15(1)$ \\
\hline & & & & S15 & & \\
\hline
\end{tabular}




\begin{tabular}{lcccccc}
$\mathrm{C}(20)$ & $54(2)$ & $38(2)$ & $114(3)$ & $-10(2)$ & $25(2)$ & $5(1)$ \\
$\mathrm{C}(21)$ & $52(2)$ & $66(2)$ & $44(2)$ & $-21(2)$ & $7(1)$ & $9(2)$ \\
$\mathrm{C}(22)$ & $57(2)$ & $70(2)$ & $33(1)$ & $12(1)$ & $10(1)$ & $-11(2)$ \\
$\mathrm{C}(23)$ & $58(2)$ & $34(2)$ & $76(2)$ & $6(1)$ & $3(2)$ & $-13(1)$ \\
$\mathrm{C}(24)$ & $37(1)$ & $69(2)$ & $48(2)$ & $12(1)$ & $17(1)$ & $4(1)$ \\
$\mathrm{C}(25)$ & $35(2)$ & $86(3)$ & $136(4)$ & $-63(3)$ & $9(2)$ & $-4(2)$ \\
$\mathrm{C}(26)$ & $45(2)$ & $63(2)$ & $79(2)$ & $-23(2)$ & $4(2)$ & $-8(1)$ \\
$\mathrm{C}(27)$ & $58(2)$ & $43(2)$ & $106(3)$ & $-17(2)$ & $-4(2)$ & $-3(2)$ \\
$\mathrm{C}(28)$ & $56(2)$ & $68(2)$ & $120(3)$ & $-58(2)$ & $-11(2)$ & $12(2)$ \\
$\mathrm{C}(29)$ & $108(3)$ & $76(3)$ & $72(2)$ & $17(2)$ & $47(2)$ & $37(2)$ \\
$\mathrm{C}(30)$ & $188(6)$ & $87(3)$ & $76(3)$ & $32(2)$ & $61(3)$ & $70(3)$ \\
$\mathrm{C}(31)$ & $148(5)$ & $89(3)$ & $102(3)$ & $29(3)$ & $72(3)$ & $66(3)$ \\
$\mathrm{C}(32)$ & $59(2)$ & $55(2)$ & $58(2)$ & $-3(1)$ & $23(2)$ & $13(2)$ \\
& & & & & & \\
\hline
\end{tabular}


Table S5. Hydrogen coordinates $\left(\times 10^{4}\right)$ and isotropic displacement parameters $\left(\AA^{2} \times 10^{3}\right)$ for 5

\begin{tabular}{|c|c|c|c|c|}
\hline & $\mathrm{X}$ & $\mathrm{y}$ & $\mathrm{z}$ & $\mathrm{U}(\mathrm{eq})$ \\
\hline $\mathrm{H}(1 \mathrm{~A})$ & 524 & 3467 & 3019 & 57 \\
\hline $\mathrm{H}(1 \mathrm{~B})$ & 375 & 2837 & 3650 & 57 \\
\hline $\mathrm{H}(1 \mathrm{C})$ & -44 & 3494 & 3358 & 57 \\
\hline $\mathrm{H}(2 \mathrm{~A})$ & 415 & 1161 & 449 & 63 \\
\hline $\mathrm{H}(2 \mathrm{~B})$ & 134 & 1882 & 791 & 63 \\
\hline $\mathrm{H}(2 \mathrm{C})$ & -224 & 1252 & 279 & 63 \\
\hline $\mathrm{H}(3 \mathrm{~A})$ & -294 & 35 & 863 & 76 \\
\hline $\mathrm{H}(3 \mathrm{~B})$ & -59 & -110 & 1810 & 76 \\
\hline $\mathrm{H}(3 \mathrm{C})$ & 339 & -52 & 1128 & 76 \\
\hline $\mathrm{H}(5)$ & 1244 & 554 & 4314 & 52 \\
\hline $\mathrm{H}(6)$ & 1377 & 542 & 5746 & 63 \\
\hline $\mathrm{H}(7)$ & 1576 & 1648 & 6487 & 67 \\
\hline $\mathrm{H}(8)$ & 1624 & 2776 & 5768 & 71 \\
\hline $\mathrm{H}(9)$ & 1471 & 2800 & 4339 & 55 \\
\hline $\mathrm{H}(11)$ & 2142 & 2718 & 3454 & 45 \\
\hline $\mathrm{H}(12)$ & 2450 & 3839 & 2942 & 57 \\
\hline $\mathrm{H}(13)$ & 1993 & 4385 & 1752 & 56 \\
\hline $\mathrm{H}(14)$ & 1230 & 3799 & 1072 & 48 \\
\hline $\mathrm{H}(15)$ & 906 & 2708 & 1603 & 39 \\
\hline $\mathrm{H}(17 \mathrm{~A})$ & 2441 & 590 & 2737 & 83 \\
\hline $\mathrm{H}(17 \mathrm{~B})$ & 2238 & 877 & 3569 & 83 \\
\hline $\mathrm{H}(17 \mathrm{C})$ & 2362 & 1471 & 2883 & 83 \\
\hline $\mathrm{H}(18 \mathrm{~A})$ & 1844 & 651 & 1407 & 64 \\
\hline $\mathrm{H}(18 \mathrm{~B})$ & 1778 & 1537 & 1542 & 64 \\
\hline $\mathrm{H}(18 \mathrm{C})$ & 1258 & 1013 & 1361 & 64 \\
\hline $\mathrm{H}(19 \mathrm{~A})$ & 1054 & 81 & 2416 & 87 \\
\hline $\mathrm{H}(19 \mathrm{~B})$ & 1452 & -1 & 3262 & 87 \\
\hline $\mathrm{H}(19 \mathrm{C})$ & 1645 & -249 & 2409 & 87 \\
\hline $\mathrm{H}(20 \mathrm{~A})$ & 4663 & 3439 & 3139 & 101 \\
\hline $\mathrm{H}(20 \mathrm{~B})$ & 4255 & 2798 & 2762 & 101 \\
\hline $\mathrm{H}(20 \mathrm{C})$ & 4442 & 3438 & 2174 & 101 \\
\hline
\end{tabular}




\begin{tabular}{|c|c|c|c|c|}
\hline $\mathrm{H}(21 \mathrm{~A})$ & 4655 & 728 & 158 & 81 \\
\hline $\mathrm{H}(21 \mathrm{~B})$ & 4846 & 306 & 1013 & 81 \\
\hline $\mathrm{H}(21 \mathrm{C})$ & 5275 & 733 & 542 & 81 \\
\hline $\mathrm{H}(22 \mathrm{~A})$ & 5303 & 2057 & 510 & 79 \\
\hline $\mathrm{H}(22 \mathrm{~B})$ & 4840 & 2533 & 851 & 79 \\
\hline $\mathrm{H}(22 \mathrm{C})$ & 4699 & 2009 & 55 & 79 \\
\hline $\mathrm{H}(23 \mathrm{~A})$ & 4328 & -79 & 3034 & 85 \\
\hline $\mathrm{H}(23 \mathrm{~B})$ & 4520 & -44 & 2141 & 85 \\
\hline $\mathrm{H}(23 \mathrm{C})$ & 3893 & -47 & 2223 & 85 \\
\hline $\mathrm{H}(24 \mathrm{~A})$ & 3513 & 1061 & 2843 & 75 \\
\hline $\mathrm{H}(24 \mathrm{~B})$ & 3903 & 1752 & 3127 & 75 \\
\hline $\mathrm{H}(24 \mathrm{C})$ & 3976 & 969 & 3612 & 75 \\
\hline $\mathrm{H}(25 \mathrm{~A})$ & 2860 & 1092 & 458 & 103 \\
\hline$H(25 B)$ & 2901 & 552 & 1251 & 103 \\
\hline $\mathrm{H}(26 \mathrm{~A})$ & 2543 & 194 & -379 & 75 \\
\hline $\mathrm{H}(26 \mathrm{~B})$ & 2410 & -227 & 442 & 75 \\
\hline $\mathrm{H}(27 \mathrm{~A})$ & 3086 & -1008 & 439 & 84 \\
\hline $\mathrm{H}(27 \mathrm{~B})$ & 3093 & -747 & -501 & 84 \\
\hline $\mathrm{H}(28 \mathrm{~A})$ & 3866 & -516 & 721 & 100 \\
\hline H(28B) & 3825 & -139 & -178 & 100 \\
\hline $\mathrm{H}(29 \mathrm{~A})$ & 3938 & 2677 & 233 & 99 \\
\hline H(29B) & 3340 & 2400 & -99 & 99 \\
\hline $\mathrm{H}(30 \mathrm{~A})$ & 3134 & 3553 & 18 & 136 \\
\hline H(30B) & 3667 & 3729 & 650 & 136 \\
\hline $\mathrm{H}(31 \mathrm{~A})$ & 2676 & 3366 & 1019 & 130 \\
\hline H(31B) & 3183 & 3669 & 1636 & 130 \\
\hline $\mathrm{H}(32 \mathrm{~A})$ & 3289 & 2533 & 2148 & 67 \\
\hline H(32B) & 2826 & 2212 & 1459 & 67 \\
\hline
\end{tabular}


Table S6. Crystal data and structure refinement for $\left(\mathrm{Me}_{2} \mathrm{~N}\right)_{3} \mathrm{Hf}-\mathrm{Si}\left(\mathrm{SiMe}_{3}\right)_{3}(\mathbf{9 b})$

\begin{tabular}{|c|c|c|}
\hline Empirical formula & \multicolumn{2}{|l|}{$\mathrm{C}_{15} \mathrm{H}_{45} \mathrm{Hf} \mathrm{N}_{3} \mathrm{Si}_{4}$} \\
\hline Formula weight & \multicolumn{2}{|l|}{558.38} \\
\hline Temperature & \multicolumn{2}{|l|}{ 173(2) K } \\
\hline Wavelength & \multicolumn{2}{|l|}{$0.71073 \AA$} \\
\hline Crystal system & \multicolumn{2}{|l|}{ Rhombohedral } \\
\hline Space group & \multicolumn{2}{|l|}{$R 3 \mathrm{c}$} \\
\hline \multirow[t]{3}{*}{ Unit cell dimensions } & $a=15.483(5) \AA$ & $\alpha=90^{\circ}$ \\
\hline & $b=15.483(5) \AA$ & $\beta=90^{\circ}$ \\
\hline & $c=19.378(8) \AA$ & $\gamma=120^{\circ}$ \\
\hline Volume & \multicolumn{2}{|l|}{ 4023(2) $\AA^{3}$} \\
\hline Z & \multicolumn{2}{|l|}{6} \\
\hline Density (calculated) & \multicolumn{2}{|l|}{$1.383 \mathrm{~g} / \mathrm{cm}^{3}$} \\
\hline Absorption coefficient & \multicolumn{2}{|l|}{$4.072 \mathrm{~mm}^{-1}$} \\
\hline$F(000)$ & \multicolumn{2}{|c|}{1704} \\
\hline Crystal size & \multicolumn{2}{|c|}{$0.45 \times 0.40 \times 0.15 \mathrm{~mm}^{3}$} \\
\hline Theta range for data collection & \multicolumn{2}{|c|}{2.59 to $28.35^{\circ}$} \\
\hline Index ranges & \multicolumn{2}{|c|}{$-20 \leq h \leq 19,-19 \leq k \leq 20,-25 \leq l \leq 25$} \\
\hline Reflections collected & \multicolumn{2}{|c|}{11001} \\
\hline Independent reflections & \multicolumn{2}{|c|}{$2027[R(\mathrm{int})=0.0348]$} \\
\hline Completeness to theta $=28.35^{\circ}$ & \multicolumn{2}{|c|}{$94.5 \%$} \\
\hline Absorption correction & \multicolumn{2}{|c|}{ Semi-empirical from equivalents } \\
\hline Max. and min. transmission & \multicolumn{2}{|c|}{0.5803 and 0.2616} \\
\hline Refinement method & \multicolumn{2}{|c|}{ Full-matrix least-squares on $F^{2}$} \\
\hline Data / restraints / parameters & \multicolumn{2}{|c|}{$2027 / 1 / 75$} \\
\hline Goodness-of-fit on $F^{2}$ & \multicolumn{2}{|l|}{1.178} \\
\hline Final R indices $[I>2 \operatorname{sigma}(I)]$ & \multicolumn{2}{|c|}{$R 1=0.0490, \mathrm{w} R 2=0.1350$} \\
\hline $\mathrm{R}$ indices (all data) & \multicolumn{2}{|c|}{$R 1=0.0567, \mathrm{w} R 2=0.1655$} \\
\hline Absolute structure parameter & \multicolumn{2}{|c|}{$0.89(4)$} \\
\hline Largest diff. peak and hole & \multicolumn{2}{|c|}{2.792 and -1.690 e. $\AA^{-3}$} \\
\hline
\end{tabular}


Table S7. Atomic coordinates $\left(\times 10^{4}\right)$ and equivalent isotropic displacement parameters $\left(\AA^{2} \times\right.$ $10^{3}$ ) for $\mathbf{9 b}$. $\mathrm{U}(\mathrm{eq})$ is defined as one third of the trace of the orthogonalized $\mathrm{U}^{\mathrm{ij}}$ tensor.

\begin{tabular}{lllll}
\hline & $\mathrm{x}$ & $\mathrm{y}$ & $\mathrm{z}$ & $\mathrm{U}(\mathrm{eq})$ \\
\hline $\mathrm{C}(1)$ & $2183(9)$ & $2277(8)$ & $3890(6)$ & $55(3)$ \\
$\mathrm{C}(2)$ & $1567(9)$ & $1341(9)$ & $5257(5)$ & $47(3)$ \\
$\mathrm{C}(3)$ & $2301(11)$ & $453(9)$ & $4241(8)$ & $68(4)$ \\
$\mathrm{C}(4)$ & $1884(8)$ & $1964(8)$ & $1822(7)$ & $52(3)$ \\
$\mathrm{C}(5)$ & $494(10)$ & $2020(9)$ & $2292(7)$ & $48(3)$ \\
$\mathrm{Hf}(1)$ & 0 & 0 & $2454(1)$ & $20(1)$ \\
$\mathrm{N}(1)$ & $926(7)$ & $1415(6)$ & $2147(4)$ & $28(1)$ \\
$\mathrm{Si}(1)$ & 0 & 0 & $3870(3)$ & $18(1)$ \\
$\mathrm{Si}(2)$ & $1582(2)$ & $1034(2)$ & $4331(2)$ & $33(1)$ \\
\hline
\end{tabular}


Table S8. Bond lengths $(\AA)$ and angles $\left(^{\circ}\right)$ for $\mathbf{9 b .}$

\begin{tabular}{|c|c|c|c|}
\hline $\mathrm{C}(1)-\operatorname{Si}(2)$ & $1.874(12)$ & $\mathrm{Hf}(1)-\mathrm{N}(1) \# 1$ & $2.017(8)$ \\
\hline$C(2)-\operatorname{Si}(2)$ & $1.859(11)$ & $\mathrm{Hf}(1)-\mathrm{N}(1) \# 2$ & $2.017(8)$ \\
\hline C(3)-Si(2) & $1.754(15)$ & $\operatorname{Hf}(1)-\mathrm{Si}(1)$ & $2.743(6)$ \\
\hline $\mathrm{C}(4)-\mathrm{N}(1)$ & $1.435(14)$ & $\operatorname{Si}(1)-\operatorname{Si}(2)$ & $2.332(4)$ \\
\hline$C(5)-N(1)$ & $1.425(14)$ & $\operatorname{Si}(1)-\operatorname{Si}(2) \# 2$ & $2.332(4)$ \\
\hline $\mathrm{Hf}(1)-\mathrm{N}(1)$ & $2.017(8)$ & $\operatorname{Si}(1)-\operatorname{Si}(2) \# 1$ & $2.332(4)$ \\
\hline $\mathrm{N}(1)-\mathrm{Hf}(1)-\mathrm{N}(1) \# 1$ & $111.7(2)$ & $\operatorname{Si}(2) \# 2-\operatorname{Si}(1)-\operatorname{Si}(2) \# 1$ & 106.22(18) \\
\hline $\mathrm{N}(1)-\mathrm{Hf}(1)-\mathrm{N}(1) \# 2$ & $111.7(2)$ & $\mathrm{Si}(2)-\mathrm{Si}(1)-\mathrm{Hf}(1)$ & $112.55(16)$ \\
\hline $\mathrm{N}(1) \# 1-\mathrm{Hf}(1)-\mathrm{N}(1) \# 2$ & $111.7(2)$ & Si(2)\#2-Si(1)-Hf(1) & $112.55(16)$ \\
\hline N(1)-Hf(1)-Si(1) & 107.1(3) & Si(2)\#1-Si(1)-Hf(1) & $112.55(16)$ \\
\hline N(1)\#1-Hf(1)-Si(1) & 107.1(3) & $\mathrm{C}(3)-\mathrm{Si}(2)-\mathrm{C}(2)$ & $108.6(7)$ \\
\hline $\mathrm{N}(1) \# 2-\mathrm{Hf}(1)-\mathrm{Si}(1)$ & 107.1(3) & $C(3)-S i(2)-C(1)$ & 111.4(8) \\
\hline$C(5)-N(1)-C(4)$ & 112.5(9) & $C(2)-S i(2)-C(1)$ & $103.9(6)$ \\
\hline C(5)-N(1)-Hf(1) & 110.1(7) & $\mathrm{C}(3)-\mathrm{Si}(2)-\mathrm{Si}(1)$ & $109.1(4)$ \\
\hline $\mathrm{C}(4)-\mathrm{N}(1)-\mathrm{Hf}(1)$ & $137.4(8)$ & $C(2)-\operatorname{Si}(2)-\operatorname{Si}(1)$ & $113.8(4)$ \\
\hline $\operatorname{Si}(2)-\operatorname{Si}(1)-\operatorname{Si}(2) \# 2$ & 106.22(18) & $C(1)-\operatorname{Si}(2)-\operatorname{Si}(1)$ & $110.0(4)$ \\
\hline $\operatorname{Si}(2)-\operatorname{Si}(1)-\operatorname{Si}(2) \# 1$ & 106.22(18) & & \\
\hline
\end{tabular}


Table S9. Anisotropic displacement parameters $\left(\AA^{2} \times 10^{3}\right)$ for $\mathbf{9 b}$. The anisotropic displacement factor exponent takes the form: $-2 \pi^{2}\left[h^{2} a *{ }^{2} U^{11}+\ldots+2 h k a * b * U^{12}\right]$

\begin{tabular}{lllllll}
\hline & $\mathrm{U}^{11}$ & $\mathrm{U}^{22}$ & $\mathrm{U}^{33}$ & $\mathrm{U}^{23}$ & $\mathrm{U}^{13}$ & $\mathrm{U}^{12}$ \\
\hline $\mathrm{C}(1)$ & $49(7)$ & $33(5)$ & $56(7)$ & $5(5)$ & $-8(5)$ & $1(5)$ \\
$\mathrm{C}(2)$ & $46(6)$ & $51(6)$ & $28(5)$ & $-7(4)$ & $-8(4)$ & $11(5)$ \\
$\mathrm{C}(3)$ & $74(10)$ & $36(6)$ & $82(10)$ & $-30(6)$ & $-44(8)$ & $19(6)$ \\
$\mathrm{C}(4)$ & $33(5)$ & $32(5)$ & $73(8)$ & $4(5)$ & $1(5)$ & $3(4)$ \\
$\mathrm{C}(5)$ & $60(7)$ & $35(5)$ & $59(7)$ & $7(5)$ & $19(6)$ & $30(5)$ \\
$\mathrm{Hf}(1)$ & $20(1)$ & $20(1)$ & $21(1)$ & 0 & 0 & $10(1)$ \\
$\mathrm{N}(1)$ & $33(4)$ & $21(4)$ & $27(4)$ & $4(3)$ & $1(3)$ & $11(3)$ \\
$\mathrm{Si}(1)$ & $18(1)$ & $18(1)$ & $20(2)$ & 0 & 0 & $9(1)$ \\
$\mathrm{Si}(2)$ & $22(1)$ & $38(1)$ & $28(1)$ & $-1(1)$ & $-5(1)$ & $8(1)$ \\
\hline
\end{tabular}


Table S10. Hydrogen coordinates $\left(\times 10^{4}\right)$ and isotropic displacement parameters $\left(\AA^{2} \times 10^{3}\right)$ for $9 b$.

\begin{tabular}{lllll}
\hline & $\mathrm{x}$ & $\mathrm{y}$ & $\mathrm{z}$ & $\mathrm{U}(\mathrm{eq})$ \\
\hline $\mathrm{H}(1 \mathrm{~A})$ & 2806 & 2718 & 4111 & 82 \\
$\mathrm{H}(1 \mathrm{~B})$ & 1751 & 2554 & 3919 & 82 \\
$\mathrm{H}(1 \mathrm{C})$ & 2299 & 2197 & 3414 & 82 \\
$\mathrm{H}(2 \mathrm{~A})$ & 1236 & 736 & 5521 & 71 \\
$\mathrm{H}(2 \mathrm{~B})$ & 1219 & 1704 & 5309 & 71 \\
$\mathrm{H}(2 \mathrm{C})$ & 2240 & 1741 & 5419 & 71 \\
$\mathrm{H}(3 \mathrm{~A})$ & 2973 & 904 & 4384 & 101 \\
$\mathrm{H}(3 \mathrm{~B})$ & 2299 & 274 & 3766 & 101 \\
$\mathrm{H}(3 \mathrm{C})$ & 2023 & -136 & 4522 & 101 \\
$\mathrm{H}(4 \mathrm{~A})$ & 1800 & 2109 & 1353 & 78 \\
$\mathrm{H}(4 \mathrm{~B})$ & 2209 & 1574 & 1827 & 78 \\
$\mathrm{H}(4 \mathrm{C})$ & 2285 & 2577 & 2067 & 78 \\
$\mathrm{H}(5 \mathrm{~A})$ & 878 & 2498 & 2642 & 72 \\
$\mathrm{H}(5 \mathrm{~B})$ & -178 & 1606 & 2451 & 72 \\
$\mathrm{H}(5 \mathrm{C})$ & 491 & 2363 & 1880 & 72 \\
\hline
\end{tabular}


Table S11. Crystal data and structure refinement for $\left[\mathrm{Hf}\left(\mathrm{NMe}_{2}\right)_{4}\right]_{2}$

\begin{tabular}{|c|c|c|}
\hline Empirical formula & \multicolumn{2}{|l|}{$\mathrm{C}_{16} \mathrm{H}_{48} \mathrm{Hf}_{2} \mathrm{~N}_{8}$} \\
\hline Formula weight & \multicolumn{2}{|l|}{709.60} \\
\hline Temperature & \multicolumn{2}{|l|}{$173(2) \mathrm{K}$} \\
\hline Wavelength & \multicolumn{2}{|l|}{$0.71073 \AA$} \\
\hline Crystal system & \multicolumn{2}{|l|}{ Monoclinic } \\
\hline Space group & \multicolumn{2}{|l|}{$\mathrm{C} 2 / \mathrm{c}$} \\
\hline \multirow[t]{3}{*}{ Unit cell dimensions } & $a=20.429(13) \AA$ & $\alpha=90^{\circ}$ \\
\hline & $b=8.454(5) \AA$ & $\beta=112.425(10)^{\circ}$ \\
\hline & $c=15.971(10) \AA$ & $\gamma=90^{\circ}$ \\
\hline Volume & \multicolumn{2}{|l|}{$2550(3) \AA^{3}$} \\
\hline Z & \multicolumn{2}{|l|}{4} \\
\hline Density (calculated) & \multicolumn{2}{|l|}{$1.849 \mathrm{~g} / \mathrm{cm}^{3}$} \\
\hline Absorption coefficient & \multicolumn{2}{|l|}{$8.153 \mathrm{~mm}^{-1}$} \\
\hline$F(000)$ & \multicolumn{2}{|c|}{1376} \\
\hline Crystal size & \multicolumn{2}{|c|}{$0.55 \times 0.45 \times 0.07 \mathrm{~mm}^{3}$} \\
\hline Theta range for data collection & \multicolumn{2}{|c|}{2.16 to $22.50^{\circ}$} \\
\hline Index ranges & \multicolumn{2}{|c|}{$-21 \leq h \leq 21,-9 \leq k \leq 9,-17 \leq l \leq 17$} \\
\hline Reflections collected & \multicolumn{2}{|c|}{6107} \\
\hline Independent reflections & \multicolumn{2}{|c|}{$1628[R(\mathrm{int})=0.0352]$} \\
\hline Completeness to theta $=22.50^{\circ}$ & \multicolumn{2}{|c|}{$97.7 \%$} \\
\hline Absorption correction & \multicolumn{2}{|c|}{ Semi-empirical from equivalents } \\
\hline Max. and min. transmission & \multicolumn{2}{|c|}{0.5991 and 0.0940} \\
\hline Refinement method & \multicolumn{2}{|c|}{ Full-matrix least-squares on $F^{2}$} \\
\hline Data / restraints / parameters & \multicolumn{2}{|c|}{1628 / 0 / 126} \\
\hline Goodness-of-fit on $F^{2}$ & \multicolumn{2}{|l|}{1.014} \\
\hline Final $R$ indices $[I>2 \operatorname{sigma}(I)]$ & \multicolumn{2}{|c|}{$R 1=0.0358, \mathrm{w} R 2=0.0967$} \\
\hline $\mathrm{R}$ indices (all data) & \multicolumn{2}{|c|}{$R 1=0.0369, \mathrm{w} R 2=0.1000$} \\
\hline Largest diff. peak and hole & \multicolumn{2}{|c|}{2.154 and -2.286 е. $\AA^{-3}$} \\
\hline
\end{tabular}


Table S12. Atomic coordinates $\left(\times 10^{4}\right)$ and equivalent isotropic displacement parameters $\left(\AA^{2} \times\right.$ $10^{3}$ ) for $\left[\mathrm{Hf}\left(\mathrm{NMe}_{2}\right)_{4}\right]_{2}$. U(eq) is defined as one third of the trace of the orthogonalized $\mathrm{U}^{\mathrm{ij}}$ tensor.

\begin{tabular}{lllll}
\hline & $\mathrm{x}$ & $\mathrm{y}$ & $\mathrm{z}$ & $\mathrm{U}(\mathrm{eq})$ \\
\hline $\mathrm{C}(1)$ & $1440(4)$ & $5810(12)$ & $1231(6)$ & $39(2)$ \\
$\mathrm{C}(2)$ & $1062(5)$ & $8282(14)$ & $584(6)$ & $43(2)$ \\
$\mathrm{C}(3)$ & $2400(5)$ & $7385(10)$ & $3291(7)$ & $31(2)$ \\
$\mathrm{C}(4)$ & $1960(4)$ & $5527(10)$ & $4048(6)$ & $36(2)$ \\
$\mathrm{C}(5)$ & $569(4)$ & $11207(10)$ & $1869(6)$ & $35(2)$ \\
$\mathrm{C}(6)$ & $1458(4)$ & $10959(10)$ & $3356(5)$ & $33(2)$ \\
$\mathrm{C}(7)$ & $-645(5)$ & $7788(9)$ & $704(6)$ & $23(2)$ \\
$\mathrm{C}(8)$ & $-122(4)$ & $5362(9)$ & $1461(5)$ & $26(2)$ \\
$\mathrm{Hf}(1)$ & $842(1)$ & $7734(1)$ & $2431(1)$ & $14(1)$ \\
$\mathrm{N}(1)$ & $938(3)$ & $10114(9)$ & $2592(4)$ & $22(1)$ \\
$\mathrm{N}(2)$ & $1121(4)$ & $7266(7)$ & $1336(5)$ & $24(2)$ \\
$\mathrm{N}(3)$ & $1760(3)$ & $6787(9)$ & $3369(4)$ & $22(1)$ \\
$\mathrm{N}(4)$ & $-286(4)$ & $7015(8)$ & $1599(5)$ & $18(2)$ \\
\hline
\end{tabular}


Table S13. Bond lengths $(\AA)$ and angles $\left(^{\circ}\right)$ for $\left[\mathrm{Hf}\left(\mathrm{NMe}_{2}\right)_{4}\right]_{2}$.

\begin{tabular}{lcll}
\hline $\mathrm{C}(1)-\mathrm{N}(2)$ & $1.432(11)$ & $\mathrm{Hf}(1)-\mathrm{N}(1)$ & $2.028(7)$ \\
$\mathrm{C}(2)-\mathrm{N}(2)$ & $1.443(12)$ & $\mathrm{Hf}(1)-\mathrm{N}(3)$ & $2.064(6)$ \\
$\mathrm{C}(3)-\mathrm{N}(3)$ & $1.450(11)$ & $\mathrm{Hf}(1)-\mathrm{N}(2)$ & $2.074(8)$ \\
$\mathrm{C}(4)-\mathrm{N}(3)$ & $1.462(10)$ & $\mathrm{Hf}(1)-\mathrm{N}(4)$ & $2.262(7)$ \\
$\mathrm{C}(5)-\mathrm{N}(1)$ & $1.447(11)$ & $\mathrm{Hf}(1)-\mathrm{N}(4) \# 1$ & $2.324(7)$ \\
$\mathrm{C}(6)-\mathrm{N}(1)$ & $1.463(10)$ & $\mathrm{Hf}(1)-\mathrm{Hf}(1) \# 1$ & $3.532(2)$ \\
$\mathrm{C}(7)-\mathrm{N}(4)$ & $1.486(11)$ & $\mathrm{N}(4)-\mathrm{Hf}(1) \# 1$ & $2.324(7)$ \\
$\mathrm{C}(8)-\mathrm{N}(4)$ & $1.474(10)$ & & \\
& & & \\
$\mathrm{N}(1)-\mathrm{Hf}(1)-\mathrm{N}(3)$ & $106.1(3)$ & $\mathrm{C}(5)-\mathrm{N}(1)-\mathrm{C}(6)$ & $110.2(7)$ \\
$\mathrm{N}(1)-\mathrm{Hf}(1)-\mathrm{N}(2)$ & $104.6(2)$ & $\mathrm{C}(5)-\mathrm{N}(1)-\mathrm{Hf}(1)$ & $122.5(5)$ \\
$\mathrm{N}(3)-\mathrm{Hf}(1)-\mathrm{N}(2)$ & $94.1(3)$ & $\mathrm{C}(6)-\mathrm{N}(1)-\mathrm{Hf}(1)$ & $126.5(5)$ \\
$\mathrm{N}(1)-\mathrm{Hf}(1)-\mathrm{N}(4)$ & $111.6(2)$ & $\mathrm{C}(1)-\mathrm{N}(2)-\mathrm{C}(2)$ & $108.1(8)$ \\
$\mathrm{N}(3)-\mathrm{Hf}(1)-\mathrm{N}(4)$ & $139.7(3)$ & $\mathrm{C}(1)-\mathrm{N}(2)-\mathrm{Hf}(1)$ & $123.1(6)$ \\
$\mathrm{N}(2)-\mathrm{Hf}(1)-\mathrm{N}(4)$ & $90.0(3)$ & $\mathrm{C}(2)-\mathrm{N}(2)-\mathrm{Hf}(1)$ & $128.7(6)$ \\
$\mathrm{N}(1)-\mathrm{Hf}(1)-\mathrm{N}(4) \# 1$ & $102.9(2)$ & $\mathrm{C}(4)-\mathrm{N}(3)-\mathrm{C}(3)$ & $108.2(6)$ \\
$\mathrm{N}(3)-\mathrm{Hf}(1)-\mathrm{N}(4) \# 1$ & $87.4(3)$ & $\mathrm{C}(4)-\mathrm{N}(3)-\mathrm{Hf}(1)$ & $137.8(5)$ \\
$\mathrm{N}(2)-\mathrm{Hf}(1)-\mathrm{N}(4) \# 1$ & $150.9(3)$ & $\mathrm{C}(3)-\mathrm{N}(3)-\mathrm{Hf}(1)$ & $113.7(5)$ \\
$\mathrm{N}(4)-\mathrm{Hf}(1)-\mathrm{N}(4) \# 1$ & $71.0(3)$ & $\mathrm{C}(7)-\mathrm{N}(4)-\mathrm{C}(8)$ & $109.3(6)$ \\
$\mathrm{N}(1)-\mathrm{Hf}(1)-\mathrm{Hf}(1) \# 1$ & $92.36(14)$ & $\mathrm{C}(7)-\mathrm{N}(4)-\mathrm{Hf}(1)$ & $117.7(5)$ \\
$\mathrm{N}(3)-\mathrm{Hf}(1)-\mathrm{Hf}(1) \# 1$ & $126.32(18)$ & $\mathrm{C}(8)-\mathrm{N}(4)-\mathrm{Hf}(1)$ & $95.9(4)$ \\
$\mathrm{N}(2)-\mathrm{Hf}(1)-\mathrm{Hf}(1) \# 1$ & $129.8(2)$ & $\mathrm{C}(7)-\mathrm{N}(4)-\mathrm{Hf}(1) \# 1$ & $109.8(5)$ \\
$\mathrm{N}(4)-\mathrm{Hf}(1)-\mathrm{Hf}(1) \# 1$ & $40.29(18)$ & $\mathrm{C}(8)-\mathrm{N}(4)-\mathrm{Hf}(1) \# 1$ & $123.0(5)$ \\
$\mathrm{N}(4) \# 1-\mathrm{Hf}(1)-\mathrm{Hf}(1) \# 1$ & $39.00(17)$ & $\mathrm{Hf}(1)-\mathrm{N}(4)-\mathrm{Hf}(1) \# 1$ & $100.7(3)$ \\
& & &
\end{tabular}

Symmetry transformations used to generate equivalent atoms:

$\# 1-\mathrm{x}, \mathrm{y},-\mathrm{z}+1 / 2$ 
Table S14. Anisotropic displacement parameters $\left(\AA^{2} \times 10^{3}\right)$ for $\left[\mathrm{Hf}\left(\mathrm{NMe}_{2}\right)_{4}\right]_{2}$. The anisotropic displacement factor exponent takes the form: $-2 \pi^{2}\left[h^{2} a^{* 2} U^{11}+\ldots+2 h k a * b * U^{12}\right]$

\begin{tabular}{lllllll}
\hline & $\mathrm{U}^{11}$ & $\mathrm{U}^{22}$ & $\mathrm{U}^{33}$ & $\mathrm{U}^{23}$ & $\mathrm{U}^{13}$ & $\mathrm{U}^{12}$ \\
\hline $\mathrm{C}(1)$ & $43(4)$ & $43(6)$ & $32(5)$ & $-11(4)$ & $16(4)$ & $13(4)$ \\
$\mathrm{C}(2)$ & $46(5)$ & $55(6)$ & $36(5)$ & $8(5)$ & $23(4)$ & $-1(5)$ \\
$\mathrm{C}(3)$ & $21(5)$ & $43(5)$ & $24(5)$ & $9(4)$ & $2(4)$ & $-1(3)$ \\
$\mathrm{C}(4)$ & $28(4)$ & $32(5)$ & $42(5)$ & $18(4)$ & $5(3)$ & $10(4)$ \\
$\mathrm{C}(5)$ & $43(4)$ & $19(4)$ & $42(5)$ & $10(4)$ & $16(4)$ & $6(4)$ \\
$\mathrm{C}(6)$ & $46(5)$ & $24(5)$ & $31(4)$ & $-9(4)$ & $17(4)$ & $-6(4)$ \\
$\mathrm{C}(7)$ & $34(5)$ & $30(5)$ & $8(4)$ & $1(3)$ & $9(4)$ & $2(3)$ \\
$\mathrm{C}(8)$ & $29(4)$ & $17(4)$ & $30(4)$ & $-9(4)$ & $8(3)$ & $-4(3)$ \\
$\mathrm{H} f(1)$ & $21(1)$ & $9(1)$ & $12(1)$ & $0(1)$ & $6(1)$ & $0(1)$ \\
$\mathrm{N}(1)$ & $26(3)$ & $18(4)$ & $23(3)$ & $-4(3)$ & $10(2)$ & $-3(3)$ \\
$\mathrm{N}(2)$ & $28(4)$ & $29(4)$ & $14(4)$ & $3(3)$ & $8(3)$ & $-2(3)$ \\
$\mathrm{N}(3)$ & $19(3)$ & $25(3)$ & $18(3)$ & $1(3)$ & $5(2)$ & $1(3)$ \\
$\mathrm{N}(4)$ & $26(4)$ & $9(3)$ & $17(4)$ & $-2(3)$ & $8(3)$ & $5(3)$ \\
\hline
\end{tabular}


Table S15. Hydrogen coordinates $\left(\times 10^{4}\right)$ and isotropic displacement parameters $\left(\AA^{2} \times 10^{3}\right)$ for $\left[\mathrm{Hf}\left(\mathrm{NMe}_{2}\right)_{4}\right]_{2}$.

\begin{tabular}{|c|c|c|c|c|}
\hline & $\mathrm{x}$ & $\mathrm{y}$ & $\mathrm{z}$ & $\mathrm{U}(\mathrm{eq})$ \\
\hline $\mathrm{H}(1 \mathrm{~A})$ & 1141 & 5305 & 661 & 59 \\
\hline $\mathrm{H}(1 \mathrm{~B})$ & 1489 & 5105 & 1739 & 59 \\
\hline $\mathrm{H}(1 \mathrm{C})$ & 1908 & 6025 & 1222 & 59 \\
\hline $\mathrm{H}(2 \mathrm{~A})$ & 1523 & 8354 & 530 & 65 \\
\hline $\mathrm{H}(2 \mathrm{~B})$ & 912 & 9339 & 689 & 65 \\
\hline $\mathrm{H}(2 \mathrm{C})$ & 712 & 7843 & 25 & 65 \\
\hline $\mathrm{H}(3 \mathrm{~A})$ & 2725 & 7769 & 3883 & 47 \\
\hline $\mathrm{H}(3 \mathrm{~B})$ & 2279 & 8256 & 2853 & 47 \\
\hline $\mathrm{H}(3 \mathrm{C})$ & 2628 & 6535 & 3085 & 47 \\
\hline $\mathrm{H}(4 \mathrm{~A})$ & 2173 & 4650 & 3838 & 54 \\
\hline $\mathrm{H}(4 \mathrm{~B})$ & 1539 & 5151 & 4138 & 54 \\
\hline $\mathrm{H}(4 \mathrm{C})$ & 2304 & 5936 & 4621 & 54 \\
\hline $\mathrm{H}(5 \mathrm{~A})$ & 345 & 12035 & 2098 & 52 \\
\hline $\mathrm{H}(5 \mathrm{~B})$ & 204 & 10637 & 1375 & 52 \\
\hline$H(5 C)$ & 906 & 11689 & 1643 & 52 \\
\hline $\mathrm{H}(6 \mathrm{~A})$ & 1819 & 11430 & 3167 & 50 \\
\hline $\mathrm{H}(6 \mathrm{~B})$ & 1685 & 10218 & 3855 & 50 \\
\hline $\mathrm{H}(6 \mathrm{C})$ & 1220 & 11796 & 3558 & 50 \\
\hline $\mathrm{H}(7 \mathrm{~A})$ & -333 & 7756 & 365 & 35 \\
\hline $\mathrm{H}(7 \mathrm{~B})$ & -751 & 8891 & 793 & 35 \\
\hline $\mathrm{H}(7 \mathrm{C})$ & -1086 & 7227 & 363 & 35 \\
\hline $\mathrm{H}(8 \mathrm{~A})$ & -527 & 4687 & 1403 & 40 \\
\hline $\mathrm{H}(8 \mathrm{~B})$ & 295 & 5012 & 1980 & 40 \\
\hline $\mathrm{H}(8 \mathrm{C})$ & -24 & 5286 & 907 & 40 \\
\hline
\end{tabular}

